\title{
Weight Prediction System for Nile Tilapia using Image Processing and Predictive Analysis
}

\author{
Lean Karlo S. Tolentino ${ }^{1}$, Celline P. De Pedro ${ }^{2}$, Jatt D. Icamina ${ }^{3}$, John Benjamin E. Navarro ${ }^{4}$ \\ Luigi James D. Salvacion ${ }^{5}$, Gian Carlo D. Sobrevilla ${ }^{6}$, Apolo A. Villanueva ${ }^{7}$, Timothy M. Amado ${ }^{8}$ \\ Maria Victoria C. Padilla ${ }^{9}$, Gilfred Allen M. Madrigal ${ }^{10}$ \\ Department of Electronics Engineering, Technological University of the Philippines, Manila, Philippines 1, 2, 3, 4, 5, 6, 7, 8, 9, 10 \\ University Extension Services, Technological University of the Philippines, Manila, Philippines ${ }^{1}$
}

\begin{abstract}
Fish farmers are likely to cultivate poor quality fish to accommodate the rising demands for food due to the everincreasing population. Fish growth monitoring greatly helps on producing higher quality fish products which leads to a better impact in the aquatic animal food production industry. However, monitoring through manual weighing and measuring stresses them that affects their health resulting to poorer quality or even fish kills. This paper presents a low-cost monitoring and Hough gradient method-based weight prediction system for Nile Tilapia (Oreochromis niloticus) using Raspberry $\mathrm{Pi}$ microcontroller and two low-cost USB cameras. This study aims to improve fish growth rate through monitoring the growth of the fishes with image processing eliminating the traditional way of obtaining fish measurements. By using paired t-test, the acquired values imply that the weight algorithm used to measure the weight of the fishes is accurate and acceptable to use. Growth performance of 10 Nile Tilapia was obtained in two intensive aquaculture setups - one for automated fish weighing through image processing and predictive analysis and the other setup for manual weighing. In response to weight prediction application, the growth of the fishes increased by $\mathbf{4 7 . 8 8 \%}$.
\end{abstract}

Keywords-Fish; growth; Tilapia; image processing; predictive analysis; weight prediction

\section{INTRODUCTION}

Due to the world's ever-growing population, humans need alternative food sources. With that, aquaculture is an essential contributor when it comes to food safety and daily living. It is the fastest growing sector in the food industry worldwide having its economic significance greatly increasing at the same time [1]. However, aquaculture is having difficulties in terms of production resulting on problems settling in the market [2]. In order to accommodate the growing demands for food, fish farmers tend to harvest poor quality fish. Thus, monitoring the growth of the fish helps to a great extent on producing a higher quality product.

Automated monitoring of the growth of the fish is a more convenient way of measuring and weighing it. According to the Florida and Wildlife Conservation Commission, manual measurement takes much labor and is time consuming on fish farmers as fishes are being captured and handled. Capture and handling are stressors that affect fish growth resulting on lower quality fish [3]. The quality of fish being lower is one of the issues aquaculture industry faces these days. Hence, with the advancement of technology, science is being used in various fields including the factory-farm and is applied to aquaculture especially in water quality monitoring [4]-[13] and fish growth \& detection [14]-[15]. This makes the monitoring of the system automated, reduces time consumed by the farmers and less stressful to the fishes.

The aim of this paper is to develop an aquaculture system that monitors the growth of the fishes using image processing to improve fish growth rate. The study specifically aims (1) to develop a fish weight prediction system for an intensive aquaculture setup that automatically predict the fish weight without manually weighing it, (2) to implement an Internet of Things framework for accessing the weight prediction system through an Internet-based application that displays the average length and weight to determine the growth of the fishes, and (3) to assess the efficiency and reliability of the system, and the difference in the rate of fish growth between the aquaculture setup with weight prediction system and the conventional setup which involves manual weighing.

The system focuses on monitoring the growth of the fishes through image processing and predictive analysis. It utilizes two low-cost USB camera and micro-computer. Preprogrammed in the micro-computer are the tested calibration settings for the implementation of stereo-vision technology to the cameras used and the weight prediction algorithm. This method lessens the effort of fish farmers on determining the growth of the fishes while minimizing the possibilities to have a lower quality fish. Moreover, as the system utilized low cost materials, the implementation of the study also benefits fish farmers cultivating fishes on small-scale aquaculture system. The data acquisition is only once per week and is set to occur at a specific time in the day. This study is restricted to cultivating one species in an Intensive Aquaculture Setup, namely Nile Tilapia (Oreochromis niloticus). Tilapia is a fastgrowing fish and tolerant of various conditions in the aquaculture environment. Due to its growth rate, low cost of production, and inexpensive on the market, Nile Tilapia is cultivated from extensive to intensive aquaculture systems in any form.

The paper is presented as follows: Section II includes the works related to the study and their gaps. The methodology and system architecture are explained in Section III. The results and discussion are presented in Section IV. Lastly, conclusion and future work are stated in Section V. 


\section{RELATED WORKS}

Aquaculture, also known as aquafarming, is the cultivation of aquatic plants and animals under controlled or semicontrolled system [16]. It is the farming of aquatic creature such as fish, mollusks, algae and other organisms. It provides a living habitat for various aquatic creature with environment conditions optimal for their growth [17]. With the growing fish demand and declining production from fisheries, aquaculture is acquiring further significance [18].

Tilapia as shown in Fig. 1 is very versatile and is tolerant to different aquaculture environments. Tilapia are cultivated in every method from extensive to intensive aquaculture system [19]. Because of its growth rate, low production cost and affordable in the market, Tilapia is being cultured in aquaculture [20]. Moreover, they only have 5 basic needs: (1) food (2) light (3) room to Swim (4) oxygen and (5) clean water.

A study in [21] stated that approximation of fish size and weight is important in fish farming. This provides data that are essential for fish feeding and harvesting. However, manual weighing and measurement are stressful to the fish.

According to a study [3], capturing and handling causes stress. Provided by experiments, evidences are documented that when handling the fish, its blood cortisol and/or glucose levels increases which are intrinsically stressful.

A study in [22] stated that Tilapia shows isometric growth patterns whenever they are culture in an environment condition optimal for their growth. This study obtained the length-weight relationship of Nile Tilapia.

Most previous works relating to aquaculture and fisheries systems only considered to assess the effects of stress to fishes due to manual weighing and determining the length and weight relationship of Nile Tilapia. The researches and information pertaining weight prediction specifically on the genus Tilapia, species Oreochromis niloticus (Nile Tilapia) generally, have not been applied or put to practical use. To further enhance the study, it is also important to consider some of the other factors that greatly affect the growth, as well as quality, of the fishes thus, the proponents considered developing a weight prediction system for Nile Tilapia. The incorporation of weight prediction system suggests for a lesser possibility of the fishes to have a poorer quality. It also reduces the effort and time consumed by the fish farmers on determining the fish growth rate. Profound research on fish growth issues show stress as one of the major factors influencing fish growth, which is why the lack of direct interaction with fish leads to a healthier fish.

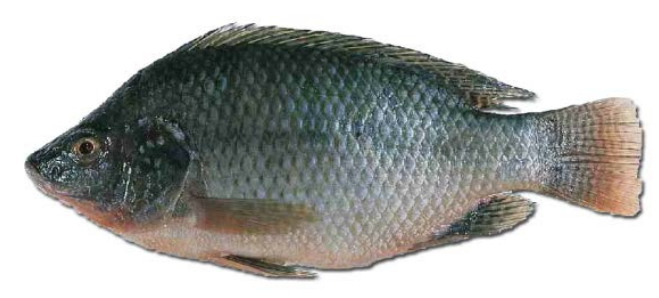

Fig. 1. Tilapia (Oreochromis Niloticus).

\section{METHODOLOGY}

\section{A. Conceptualization and Design}

The design and development of this study is mainly concerned with the weight prediction system for Nile Tilapia. Weight of the fish is the vital parameter that was considered in this study.

Fig. 2 shows the process flowchart. Two low-cost USB Camera were used. These cameras are programmed into a Stereo-Vision camera for distance measurement. The camera captured images which are processed in the Arduino Mega. The system detects fishes and acquires different parameters needed for predictive analysis. The then captured images are processed through various filters to accurately calculate the actual length of the fishes for the weight prediction through length and weight correlation. From here, the data are sent to the database via Long Range Wide Area Network (LoRaWAN) IoT Protocol and are displayed to the Web Application.

\section{B. Fish Weight Prediction System}

Two aquaculture systems as shown in Fig. 3 were made to culture 10 Nile Tilapia. The first system employs conventional method of weighing Tilapia wherein the fishes are being captured, handled and placed on a digital weighing scale while the other system employs automated fish weighing where a camera is used to predict the weight of the fish.

Fig. 4 shows the system flowchart of the proposed system. The input parameter in the system includes the image of fish. For the process it includes image processing that is composed of various sections namely: cropping which is the division of image into two regions of interest [23], grayscale used for converting image into shades of gray with no apparent color, blurring which is the relative motion between a camera that is out of focus and a specific object [24], binary thresholding to create binary images, erosion for removal of pixels on object boundaries, dilation for adding pixels on object boundaries and morphological closing for filling small holes from an image without affecting the size and shape of the objects in the image.

Two low-cost USB camera were utilized to produce stereocamera, implementing stereo-vision technology. Multiple images from both cameras were collected for calibration and depth map tuning. Tested calibration settings were loaded to attain accurate distance measurements.

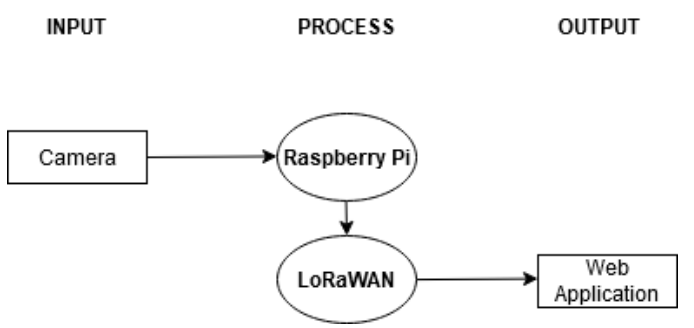

Fig. 2. Process Flowchart. 


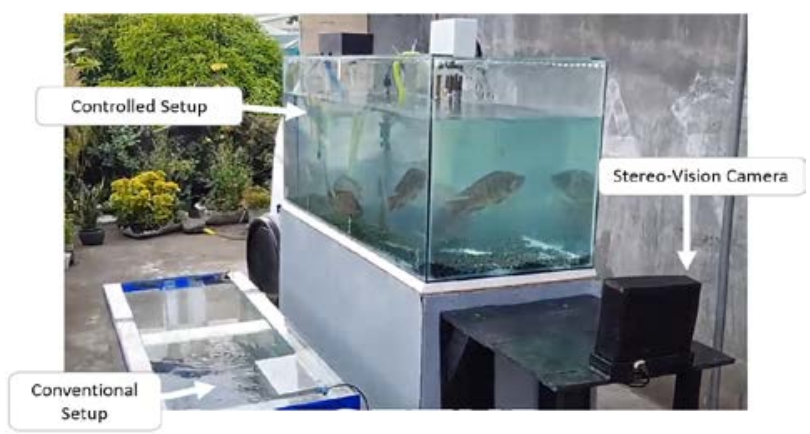

Fig. 3. Conventional and Automated Aquaculture System.

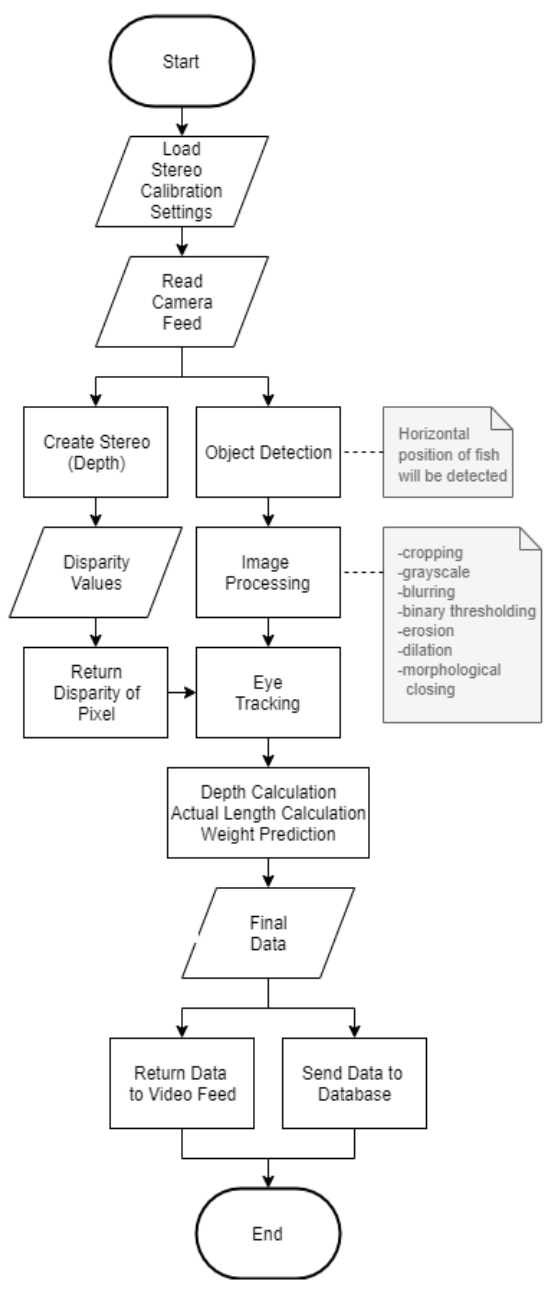

Fig. 4. System Flowchart.

After reading the camera feed, object detection and depth map creation were processed simultaneously. Frames, with detected images of Nile Tilapia, undergone series of image processing techniques to filter only the contour of fish, as well as removal of noises and unwanted data for more accurate measurements.

Once the contour was drawn, eye-tracking technique through circle detection was performed to pin-point consistent part of fish as basis of its distance from the camera. Hough Gradient Method of the OpenCV is implemented to detect circles. This method utilizes the gradient information of edges.
If a circle is parameterized by its radius $r$ and its center coordinates $(a, b)$, then these are correlated to the position of edge points $(x, y)$, which create the circle by means of the limitation:

$(x-a)^{2}+(y-b)^{2}=r^{2}$

Equation 1 also specifies that "every certain edge point $(x, y)$ could be a point on any circles" where "their limitations lie on the plane of a right circular cone in the $(a, b, r)$ parameter space." All image points which are on the circle interpreted by those three parameters if the cones corresponding to several edge points cross at an only point [24].

Actual length was measured using the contour of fish, converting its pixel length to an actual measurement following pixel/metric conversion. Lastly, actual length was used for weight prediction through predictive analysis.

The weight prediction which is derived using polynomial regression is represented as:

$w=0.1017 x^{3}-4.8944 x^{2}+93.44 x-583.06$

Where: $\mathrm{w}=$ weight of the fish predicted

$$
\mathrm{X}=\text { length measured }
$$

Fig. 5 shows the image processing once the fish is detected. Once the contour was drawn, circle detection takes place to detect the eye of the fish for basis of distance measurement.

Fig. 6 shows the actual fish detection with length measurement and weight prediction.

\section{Distance Measurement}

A stereo-vision camera has two or more lenses with a distinct image sensor for every lens and have the ability to perceive depth. Instead of using costly depth camera, two lowcost USB cameras are calibrated to act as a stereo-vision camera.

The cameras are calibrated in order to calculate the disparity value between frames captured from left and right camera as shown in Fig. 7.

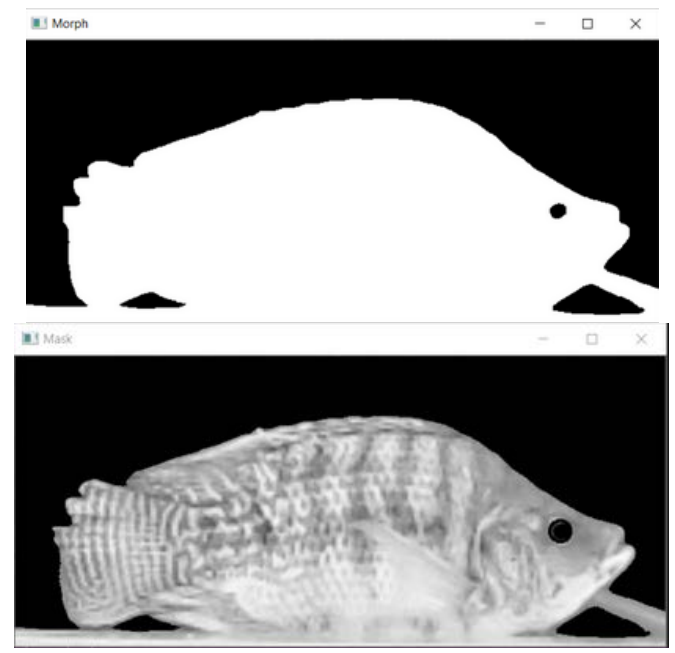

Fig. 5. Image Processing. 


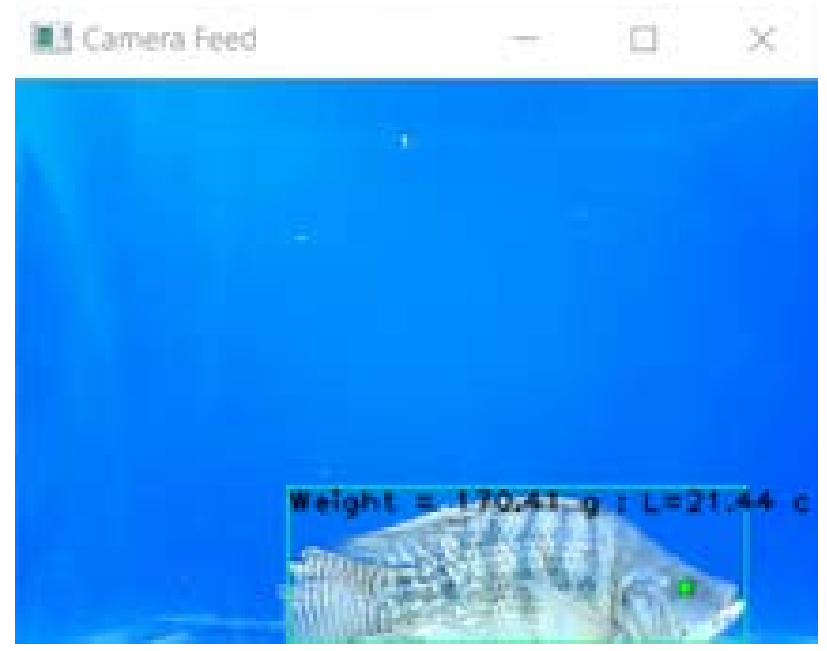

Fig. 6. Weight Prediction.

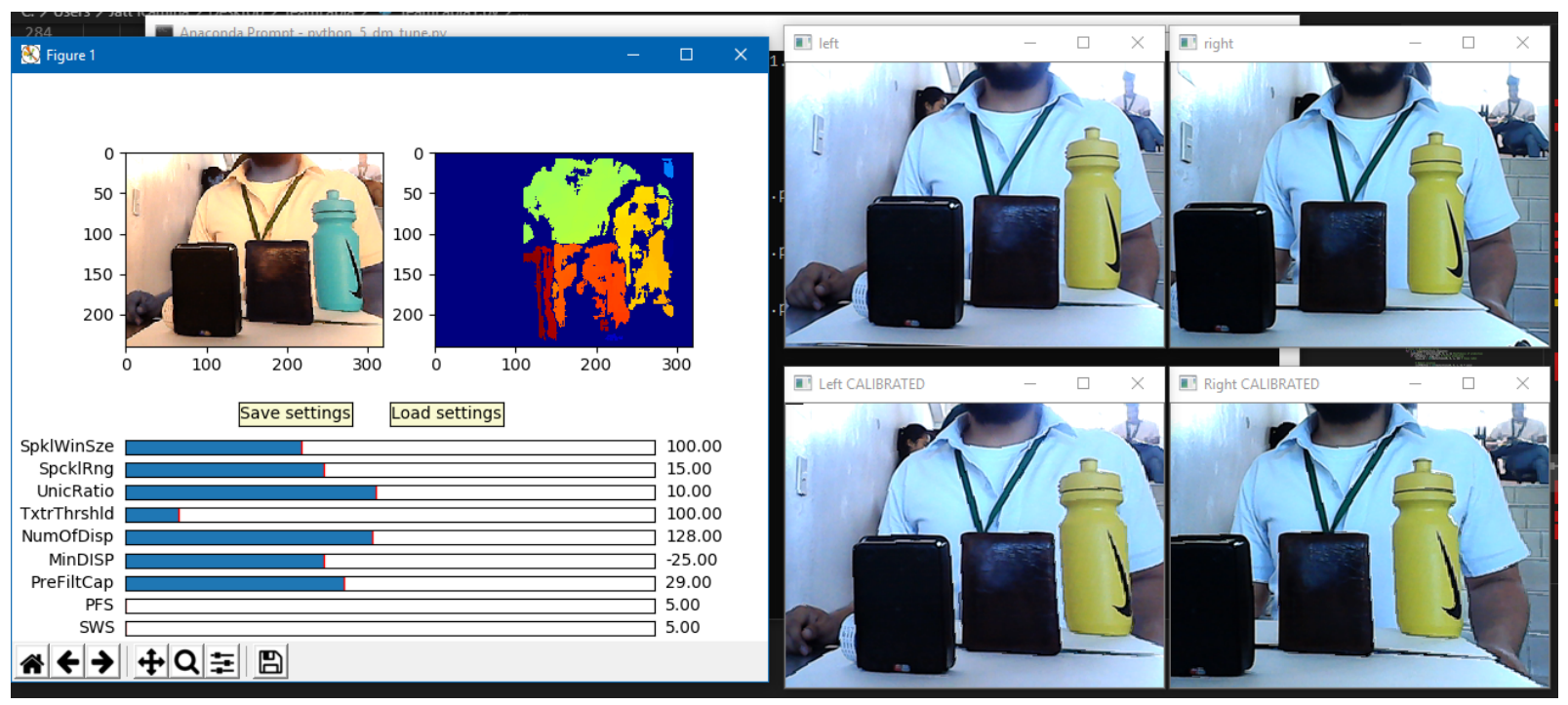

Fig. 7. Calibration Settings.

Fig. 8 shows the Regression analysis between the actual distance and disparity value. The acquired equation for the distance of the fish in terms of pixel is calculated as:

$D=0.1017 z^{3}-4.8944 z^{2}+93.44 z-583.06$

Where: $\mathrm{D}=$ distance in terms of pixel

$$
\mathrm{z}=\text { disparity value }
$$

To identify the actual distance of the fish from the camera, the obtained virtual length (in terms of pixel) is calculated following $\mathrm{pixel} / \mathrm{cm}$ conversion using regression analysis as shown in Fig. 9. The acquired actual distance is used to calculate the length of the fish that is needed for weight prediction.

The actual distance of the fish following pixel/cm conversion is represented as:

ppcm $=0.0017 z^{2}-0.1601 z-10.18$

\section{Examined Trendlines}

Fig. 10 shows the relationship between length and weight in a polynomial trendline. The acquired R2 value is 0.9324 while the equation for weight computation is $0.1017 \mathrm{x}^{3}$ $4.8944 x^{2}+93.44 x-583.06$.

\section{Actual Distance vs Disparity Value}

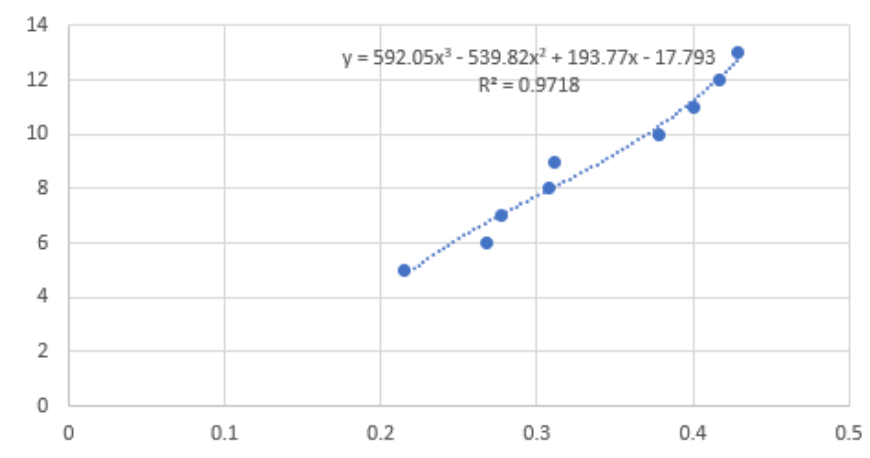

Fig. 8. Actual Distance vs Disparity Value 


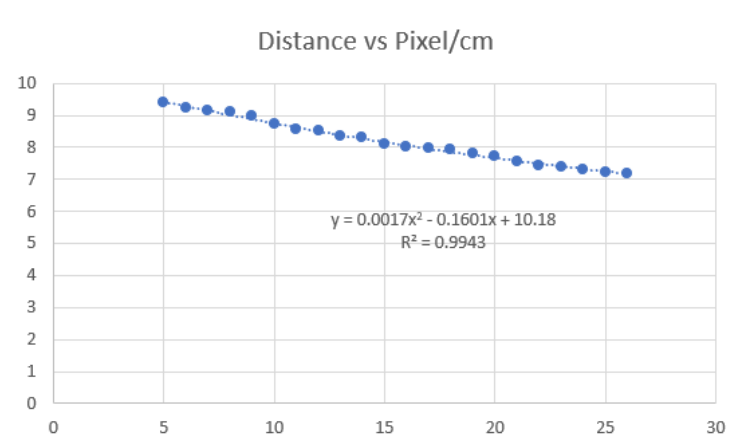

Fig. 9. Distance vs. Pixel/cm.

\section{LENGTH VS WEIGHT \\ (Polynomial)}

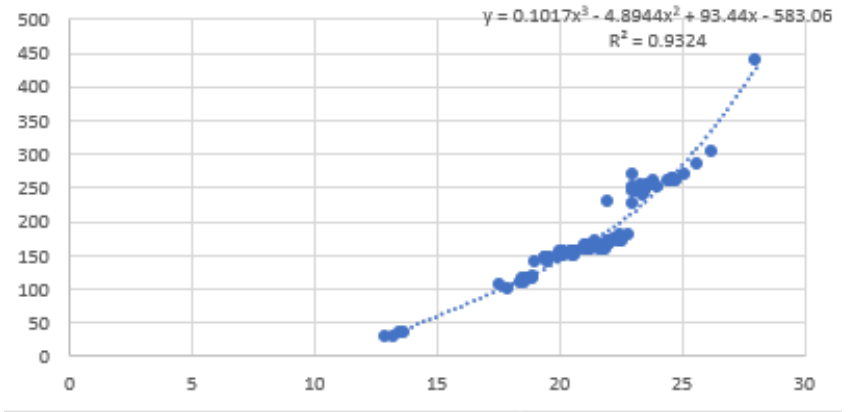

Fig. 10. Length in Cm vs. Weight in Grams (Polynomial).

Fig. 11 shows the correlation of length and weight in a Exponential trendline. The value of the R2 acquired is 0.9286 while the equation for weight computation is $4.7915 \mathrm{e}^{0.1661 \mathrm{x}}$.

Fig. 12 shows the correlation of length and weight in a Linear trendline. The value of the R2 acquired is 0.8741 while the equation for weight computation is $23.212 \mathrm{x}-315.08$.

Fig. 13 shows the correlation of length and weight in a Logarithmic trendline. The value of the R2 acquired is 0.8125 while the equation for weight computation is $435.58 \ln (\mathrm{x})$ 1149.1.

Various trendlines were examined to determine the most suitable to the data gathered such as Polynomial, Exponential, Linear and Logarithmic. The value of the R2 was used to determine the fitness of the line. The closer to the value of 1 , the best fit trendline to the dataset.

\section{E. Web Application}

The TeamLapia web application as shown in Fig. 14 is made to organize all the data gathered and display the most recent status of the fishes. The web application exhibits the numerical values of the essential parameters including the average length and weight to determine the growth of the fishes. Through the use of various JavaScript and php codes, data are transmitted from the database to the UI. On the other hand, using html, CSS and JavaScript scripts, the graphs and dynamic design of the application are made to make it more suitable for end-users.

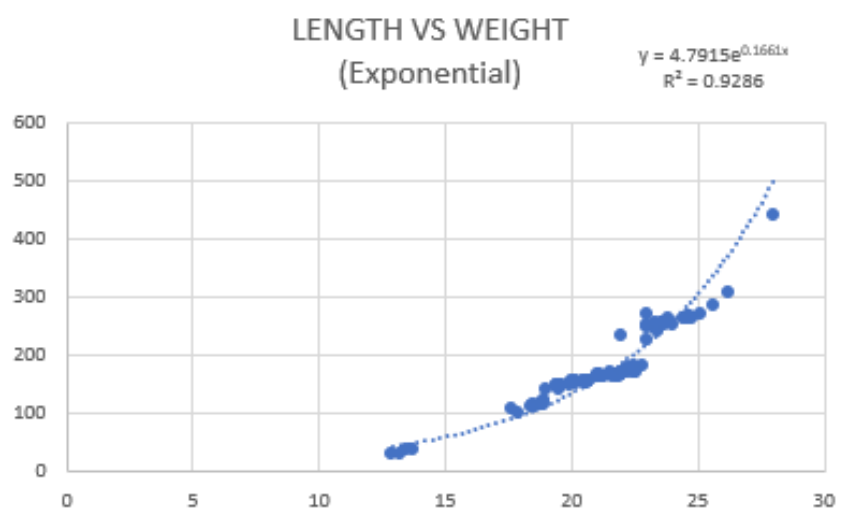

Fig. 11. Length in Cm vs. Weight in grams (Exponential).

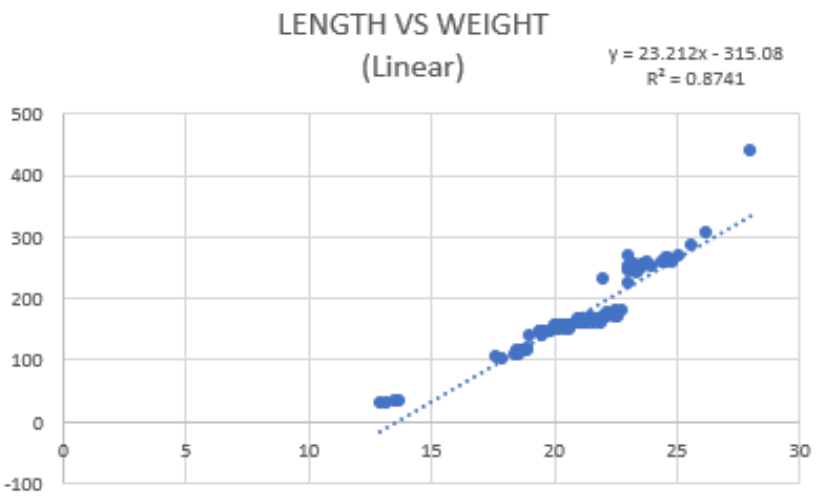

Fig. 12. Length vs Weight (Linear).

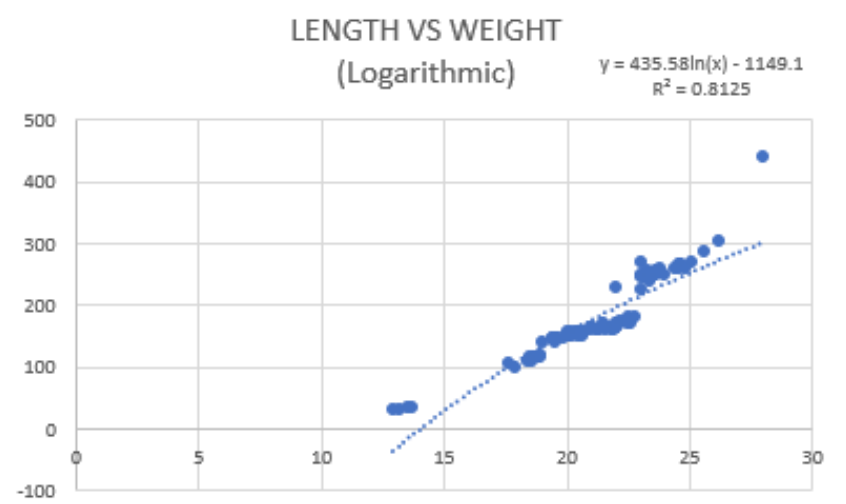

Fig. 13. Length in $\mathrm{cm}$ vs Weight in grams (Logarithmic).

\section{F. Statistical Analysis}

The results obtained between the measured and predicted weight of the samples were evaluated statistically using t-test: Paired Two Sample for Means.

The test statistic is calculated as:

$t=\frac{\bar{d}}{\sqrt{s^{2} / n}}$

Where: d' = mean difference

$$
\begin{aligned}
& \mathrm{s}^{2}=\text { sample variance } \\
& \mathrm{n}=\text { sample size }
\end{aligned}
$$

$\mathrm{t}=\mathrm{t}$ quantile with $\mathrm{n}-1$ degrees of freedom 


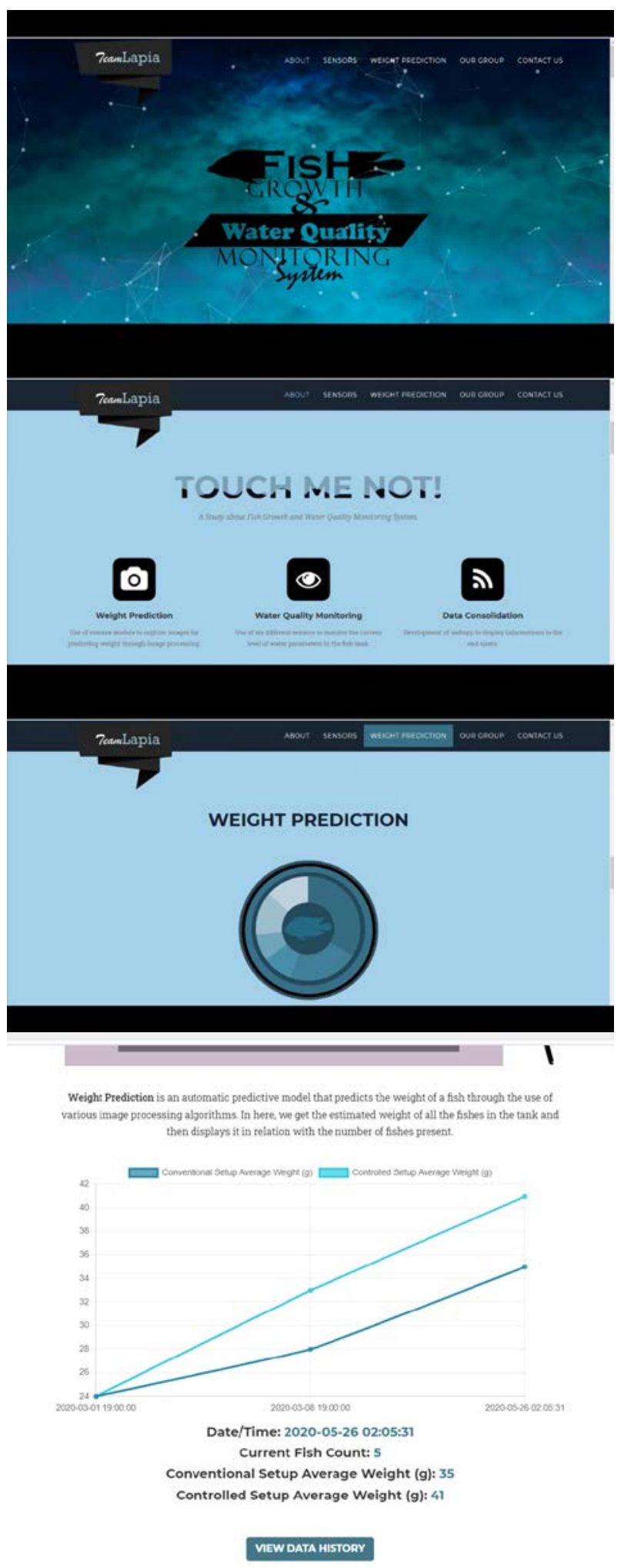

Fig. 14. Web Application Interface.

\section{RESULTS AND DISCUSSION}

To test the accuracy of the Weight Prediction Algorithm, a comparison was made between the actual measured weight of the fishes and the predicted weight. Table I shows the recorded weights of ten fish samples and their corresponding percentage error. With the data assimilated, it was inferred that the measured and predicted weight were almost the same as it only has a mean percentage error of $2.82 \%$. With that in mind, to further test the accuracy of the device, a hypothesis test was also conducted.

The p-value for two-tailed test is greater than the significance level of 0.01 which means that we have to accept the null hypothesis that there is no significant difference between the measured and predicted weight of the samples, Table II. This implies that the weight prediction algorithm used to measure the weight of the fishes is accurate and is acceptable to use.

TABLE I. EXPERIMENTAL DATA FOR PREDICTED AND MEASURE WEIGHT OF SAMPLE FISHES

\begin{tabular}{|l|l|l|l|l|}
\hline \multirow{2}{*}{$\begin{array}{l}\text { Fish } \\
\text { Sample }\end{array}$} & \multicolumn{2}{|l|}{ Weight (g) } & \multirow{2}{*}{ Difference } & Error (\%) \\
\cline { 2 - 3 } & Measured & Predicted & & \\
\hline 1 & 105.5 & 110 & -4.5 & $4.09 \%$ \\
\hline 2 & 97.5 & 105 & -7.5 & $7.14 \%$ \\
\hline 3 & 148.4 & 150 & -1.6 & $1.06 \%$ \\
\hline 4 & 141.6 & 150 & -8.4 & $5.6 \%$ \\
\hline 5 & 152.4 & 150 & 2.4 & $1.6 \%$ \\
\hline 6 & 104.7 & 105 & -0.3 & $0.28 \%$ \\
\hline 7 & 147.5 & 150 & -2.5 & $1.67 \%$ \\
\hline 8 & 134.8 & 135 & -0.2 & $0.15 \%$ \\
\hline 9 & 108.4 & 110 & -1.6 & $1.45 \%$ \\
\hline 10 & 104.3 & 110 & -5.7 & $5.18 \%$ \\
\hline \multicolumn{2}{|l|}{ Mean Percentage Error } & & $\mathbf{2 . 8 2 \%}$ \\
\hline \multicolumn{2}{|l|}{} & & \\
\hline
\end{tabular}

TABLE II. TWO SAMPLE FOR MEANS PAIRED T-TEST OF EXPERIMENTAL DATA FOR PREDICTED AND MEASURED WEIGHT OF SAMPLE FISHES

\begin{tabular}{|l|l|l|}
\hline & Measured & Predicted \\
\hline Mean & 124.51 & 127.5 \\
\hline Variance & 491.8232222 & 445.8333333 \\
\hline Observations & 10 & 10 \\
\hline Pearson Correlation & 0.988461845 & \\
\hline Hypothesized Mean Difference & 0 & \\
\hline df & 9 & \\
\hline t Stat & 2.736983041 & \\
\hline P(T<=t) one-tail & 0.011480323 & \\
\hline t critical one-tail & 2.821437925 & \\
\hline P(T<=t) two-tail & 0.022960645 & \\
\hline t critical two-tail & 3.249835542 & \\
\hline
\end{tabular}


The weight of Nile Tilapia was monitored in both conventional and automated aquaculture setup. Initially, the weight of the fishes on both setups are equal and the growth of the fishes is observed for two weeks. The fish growth rate based on weight in the automated setup which uses camera for weight prediction was $17 \mathrm{~g}$ (from $24 \mathrm{~g}$ to $41 \mathrm{~g}$ ) while the conventional system which employ manual weighing of fish only obtained an average of $11 \mathrm{~g}$ (34g to $35 \mathrm{~g}$ ) as shown in Fig. 15.

Table III shows the measured weight of the fish cultured in the automated setup which employs camera for weight prediction.

Table IV shows the measured weight of the fish cultured in the conventional setup which uses the manual way of weighing fish that involves, catching, handling and measuring on a digital weighing scale.

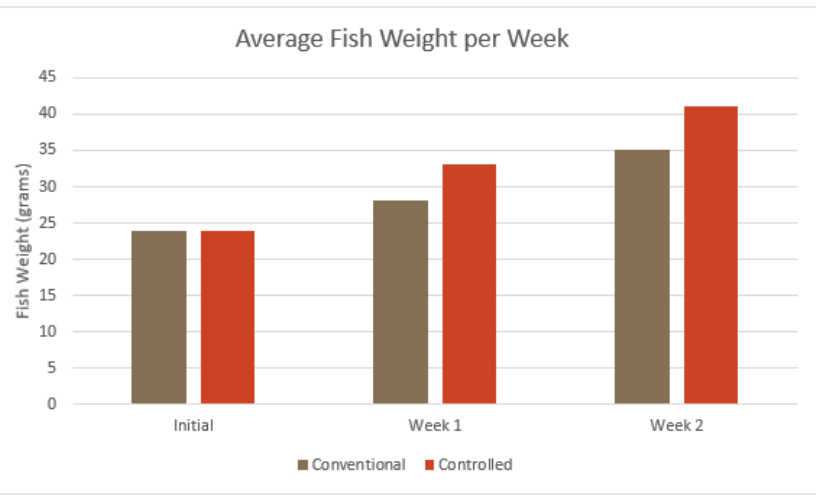

Fig. 15. Conventional vs Automated Aquaculture Setup Fish Growth measured Every Week.

TABLE III. FISH WEIGHT IN AUtOMATED SETUP MEASURED USING CAMERA

\begin{tabular}{|l|l|l|l|}
\hline Fish No. & Initial & Week 1 & Week 2 \\
\hline 1 & 20 & 25 & 35 \\
\hline 2 & 20 & 30 & 35 \\
\hline 3 & 25 & 35 & 40 \\
\hline 4 & 25 & 35 & 45 \\
\hline 5 & 30 & 40 & 50 \\
\hline Average & 24 & 33 & 41 \\
\hline
\end{tabular}

TABLE IV. Fish Weight IN CONVENTIONAL SETUP MEAsured MANUALLY

\begin{tabular}{|l|l|l|l|}
\hline Fish No. & Initial & Week 1 & Week 2 \\
\hline 1 & 20 & 25 & 30 \\
\hline 2 & 20 & 25 & 30 \\
\hline 3 & 25 & 30 & 35 \\
\hline 4 & 25 & 30 & 40 \\
\hline 5 & 30 & 35 & 40 \\
\hline Average & 24 & 28 & 35 \\
\hline
\end{tabular}

Based on the data shown, the growth rate in the automated aquaculture setup is $30.70 \%$ each week and is greater compared to the conventional setup which has $20.76 \%$ growth rate per week. The automated aquaculture setup which employs weight prediction through camera improves the growth of the fishes in terms of weight by $47.88 \%$.

\section{CONCLUSION}

The authors developed an automated aquaculture setup with weight prediction technology that makes use of image processing techniques and predictive analysis which yields to a higher growth and survival rate for Nile Tilapia. By avoiding stressors to fishes, such as capturing and handling to manually measure its weight and monitor its growth, the data gathered shows beneficent results to the rate of fish growth. The growth rate of the fishes in terms of weight improves by $47.88 \%$ with only $2.82 \%$ mean percentage error on the weight prediction. For future work, this study can be applied for growth monitoring of other animals on various environment.

For future work, this study can also be applied in predicting the weight and length of other aquatic animals in different aquaculture systems to determine its growth. This lessens the effort of the fish farmers and minimizes the possibilities of the fish to have a lower quality. Moreover, the acquired length and weight measurements may also be used to determine the number of fishes to cultivate in a given area to prevent space and food deprivation.

\section{ACKNOWLEDGMENT}

The authors would like to acknowledge Technological University of the Philippines for the partial funding and Barangay Pulanglupa Uno, Las Piñas City, Philippines which is led by Hon. Guadalupe A. Rosales for the support in the deployment and utilization of this project.

\section{REFERENCES}

[1] FAO, 2009. The state of world fisheries and aquaculture 2008. Rome, Italy.

[2] S. Han, Y. Kang, K. Park, and M. Jang, "Design of environment monitoring system for aquaculture farms," in 2007 Frontiers in the Convergence of Bioscience and Information Technologies, pp. 889-893, 2007.

[3] C. Harper and J. C. Wolf, "Morphologic effects of the stress response in fish,” ILAR Journal, vol. 50, no. 4, pp. 387-396, 2009.

[4] L. Wenmei and L. Yuzhen, “Aquaculture monitoring system," in 2010 International Forum on Information Technology and Applications, pp. 138-141, 2010.

[5] L. K. S. Tolentino et al., "Development of an IoT-based Intensive Aquaculture Monitoring System with Automatic Water Correction," International Journal of Computing and Digital Systems, in press.

[6] N. Gallah, O. B. Bahri, N. Lazreg, A. Chaouch, and K. Besbes, "Water quality monitoring based on small satellite technology," International Journal of Advanced Computer Science and Applications, vol. 8, no. 3, pp. 357-362, 2017.

[7] L. K. Tolentino et al., "IoT-Based Automated Water Monitoring and Correcting Modular Device Via LoRaWAN for Aquaculture," International Journal of Computing and Digital Systems, in press.

[8] M. A. Shareef, A. Toumi, and A. Khenchaf, "Estimation of water quality parameters using the regression model with fuzzy k-means clustering," International Journal of Advanced Computer Science and Applications, vol. 5, no. 6, pp. 151-157, 2014. 
[9] A. Maestre, Eman El-Sheikh, Derek Williamson and Amelia Ward, "A Machine Learning Tool for Weighted Regressions in Time, Discharge, and Season" International Journal of Advanced Computer Science and Applications, vol. 5, no. 3, pp. 99-106, 2014.

[10] L. K. Tolentino et al., "AquaDroid: An App for Aquaponics Control and Monitoring," in 6th International Conference on Civil Engineering (6th ICCE 2017), pp. 1-8, 2017.

[11] E. Galido et al., "Development of a Solar-powered Smart Aquaponics System through Internet of Things (IoT)," in Lecture Notes on Research and Innovation in Computer Engineering and Computer Sciences, pp. 31-39, 2019.

[12] L. K. S. Tolentino et al., "Development of an IoT-based Aquaponics Monitoring and Correction System with Temperature-Controlled Greenhouse,” 2019 International SoC Design Conference (ISOCC), 2019, pp. 261-262.

[13] L. K. S. Tolentino et al., "Yield evaluation of Brassica rapa, Lactuca sativa, and Brassica integrifolia using image processing in an IoT-based aquaponics with temperature-controlled greenhouse,” Agrivita Journal of Agricultural Science, in press.

[14] R. R. Herrera and F. G. Funes, "Affective Educational Application of Fish Tank Hydroponics System," International Journal of Advanced Computer Science and Applications, vol. 10, no. 12, pp. 126-131, 2019.

[15] K. Raza and S. Hong, "Fast and Accurate Fish Detection Design with Improved YOLO-v3 Model and Transfer Learning," International Journal of Advanced Computer Science and Applications, vol. 11, no. 2, pp. 7-16, 2020.

[16] J. H. Tidwell and L. A. Bright, "Freshwater Aquaculture," Encyclopedia of Ecology, pp. 91-96, 2018. doi: 10.1016/b978-0-12-409548-9.10618-9
[17] K. K. Kishore, P. V. Krishna, and D. Srikanth, "Automatic Feeding system for Aquaculture,” in 2017 Third International Conference on Sensing, Signal Processing and Security (ICSSS), pp. 426-429, 2017.

[18] S. N. Ojha and S. C. Babu, "Why convergence of Fisheries Comanagement with Agricultural Technology Management Agency is significant," in Agricultural Extension Reforms in South Asia, pp. 329347, 2019.

[19] K. Fitzsimmons, "Tilapia: the most important aquaculture species of the 21st century," in Proceedings from the fifth International Symposium on tilapia Aquaculture, pp. 3-8, 2000.

[20] Q. Y. Guo, X. C. Wang, X. S. Yang, Z. Xu, M. Gu, and X. Y. Li, "Freshness changes and shelf life prediction of cultured Tilapia (Oreochromis niloticus) during chilled storage," in 2011 Fourth International Conference on Intelligent Computation Technology and Automation, pp. 86-92, 2011.

[21] R. Tillett, N. McFarlane, and J. Lines, "Estimating dimensions of freeswimming fish using 3D point distribution models," Computer Vision and Image Understanding, vol. 79, no. 1, pp. 123-141, 2000.

[22] A. A. B. Adam and A. M. Khalid, "Length weight relationship and condition factor of Nile Tilapia Oreochromis niloticus (Trewavas, 1983) in the southern part of Jebel Aulia Dam, White Nile, Sudan,” Direct Res. J. Agric. Food Sci, vol. 4, no. 10, pp. 286-289, 2016.

[23] L. K. S. Tolentino, J. W. F. Orillo, P. D. Aguacito, E. J. M. Colango, J. R. H. Malit, J. T. G. Marcelino, A. C. Nadora, and A. J. D. Odeza, "Fish freshness determination through support vector machine," Journal of Telecommunication, Electronic and Computer Engineering (JTEC), vol. 9, no. 2-5, pp. 139-143, 2017.

[24] Y-. L. You and M. Kaveh, "A regularization approach to joint blur identification and image restoration," IEEE Transactions on Image Processing, vol. 5, no. 3, pp. 416-428, 1996. 